\section{ORIGINAL RESEARCH}

L.-L. Chan

H.-E. Tan

S. Fook-Chong

T.-H. Teo

L.-H. Lim

L.-L. Seah

\title{
Graves Ophthalmopathy: The Bony Orbit in Optic Neuropathy, Its Apical Angular Capacity, and Impact on Prediction of Risk
}

BACKGROUND AND PURPOSE: Optic neuropathy (ON), a serious complication of Graves ophthalmopathy, is often subclinical and masked by symptoms of orbitopathy. We examined herein bony and soft-tissue CT features associated with ON, including an angular assessment of orbital apex capacity, and their usefulness in the risk prediction of ON.

\begin{abstract}
MATERIALS AND METHODS: The CT scans of 41 patients with Graves ophthalmopathy 17 men, 24 women; mean age, 49.1 years) clinically diagnosed with (19 patients, 32 orbits) or without ON were evaluated by 2 independent raters. Quantitative linear and angular measurements of the orbital structures and bony walls and categoric scores of apical crowding and intracranial fat prolapse were assessed on a clinical workstation. Inter- and intrarater variability of these features was determined. The CT features of the 2 patient groups were compared, and multivariate logistic regression analysis was performed to evaluate the predictive features of ON.
\end{abstract}

RESULTS: Bony orbital angles $(P<.005)$, length of the lateral orbital wall $(P<.05)$, muscular diameters $(P<.0005)$, muscular bulk of the medial rectus muscle relative to the bony orbit $(P<.05)$, and apical crowding $(P<.0005)$ were associated with clinical ON. Stepwise multivariate logistic regression analysis revealed the muscle diameter index and medial and lateral wall angles to be independent predictors. Combining these in a single multivariate equation yielded sensitivity, specificity, and positive and negative predictive values of $73 \%, 90 \%, 82 \%$, and $85 \%$, respectively.

CoNCLusıons: Orbital wall angles, especially the medial wall, and muscular enlargement are independent risk predictors.

$\mathbf{G}^{\mathrm{r}}$ raves ophthalmopathy is an autoimmune inflammatory process that affects the orbital and periorbital tissue, closely associated with dysthyroidism. Approximately $6 \%$ of patients with Graves ophthalmopathy develop optic neuropathy $(\mathrm{ON})$, a potentially blinding complication. ${ }^{1}$ This is primarily attributed to apical orbital crowding and optic nerve compression. A subgroup of patients is believed to have optic nerve stretching secondary to increased orbital volume and a narrow orbital apex (particularly Asians), which may lead to earlier compressive features without other significant manifestations. ${ }^{1}$ ON is often subclinical and may be masked by other symptoms of myopathy, inflammation, and orbital congestion. ON may also occur without proptosis, and visual loss may sometimes be rapid and irreversible. ${ }^{1,2}$ Because prognosis improves with early intervention, identification of patients at risk is highly desirable. Despite improved diagnostic tests, the diagnosis of $\mathrm{ON}$ remains heavily dependent on clinical findings. ${ }^{1,3}$

Various imaging features have been associated with the diagnosis of optic neuropathy, such as the degree of enlargement of the extraocular muscles, volumetric increase in orbital fat, radiologic evidence of apical optic nerve compression, and the presence of intracranial fat prolapse. ${ }^{4-8}$ To our knowledge, the role of the bony orbit in ON has not been directly investigated. The purpose of this study was to examine both bony orbital and soft-tissue CT characteristics associated with $\mathrm{ON}$ in

Received August 22, 2008; accepted after revision October 17.

From the Departments of Diagnostic Radiology (L.-L.C., H.-E.T., T.-H.T.) and Clinical Research (S.F.-C.), Singapore General Hospital, Singapore; and Singapore National Eye Centre (L.-H.L., L.-L.S.), Singapore.

Please address correspondence to Ling-Ling Chan, MD, Department of Diagnostic Radiology, Singapore General Hospital, Outram Rd, Singapore 169608; e-mail: gdrcll@sgh.com.sg DOI 10.3174/ajnr.A1413
Graves ophthalmopathy, including a novel angular assessment of orbital apex capacity, and their usefulness in the risk prediction of $\mathrm{ON}$.

\section{Materials and Methods}

\section{Clinical}

Institutional review board approval was obtained for this study. The CT scans and medical records of 41 patients ( 17 men, 24 women) with clinically proved Graves ophthalmopathy who were referred to a single ophthalmologist in the oculoplastic service of a tertiary referral eye center during a 5-year period were collected and reviewed. The diagnostic criteria for Graves disease included clinical symptoms and signs of hyperthyroidism, high serum free thyroxine, suppressed thyroid stimulating hormone, and a positive thyrotropin-receptor antibody test. All patients had a complete standardized ophthalmologic examination performed within 4 weeks before CT. The clinical diagnosis of ON was made on the basis of the presence of $\geq 2$ of the following: reduced best corrected visual acuity, impaired color vision, visual field defect not attributable to other eye disease, afferent papillary defect, or optic disc swelling/atrophy. Nineteen patients had ON, and in 6 , the disease involved only 1 orbit ( 32 orbits). The patients ranged from 28 to 78 years of age (mean, 49.1 years).

\section{Imaging}

The 41 orbital scans were part of a routine clinical protocol performed following intravenous contrast administration on helical CT scanners in our institution, by using acquisition parameters of 120 kilovolt (peak) (kVp), $200 \mathrm{~mA}, 160-\mathrm{mm}$ display FOV, and pitch of $0.8-1.0$. The axial scans were contiguous 3 -mm sections parallel to the infraorbitomeatal line, and coronal scans were reconstructed orthogonal to this plane. Care was taken to ensure that the axial scans were indeed parallel to the infraorbitomeatal line and, when necessary, reconstructed on a $3 \mathrm{D}$ workstation from the archived fine-section acquisi- 

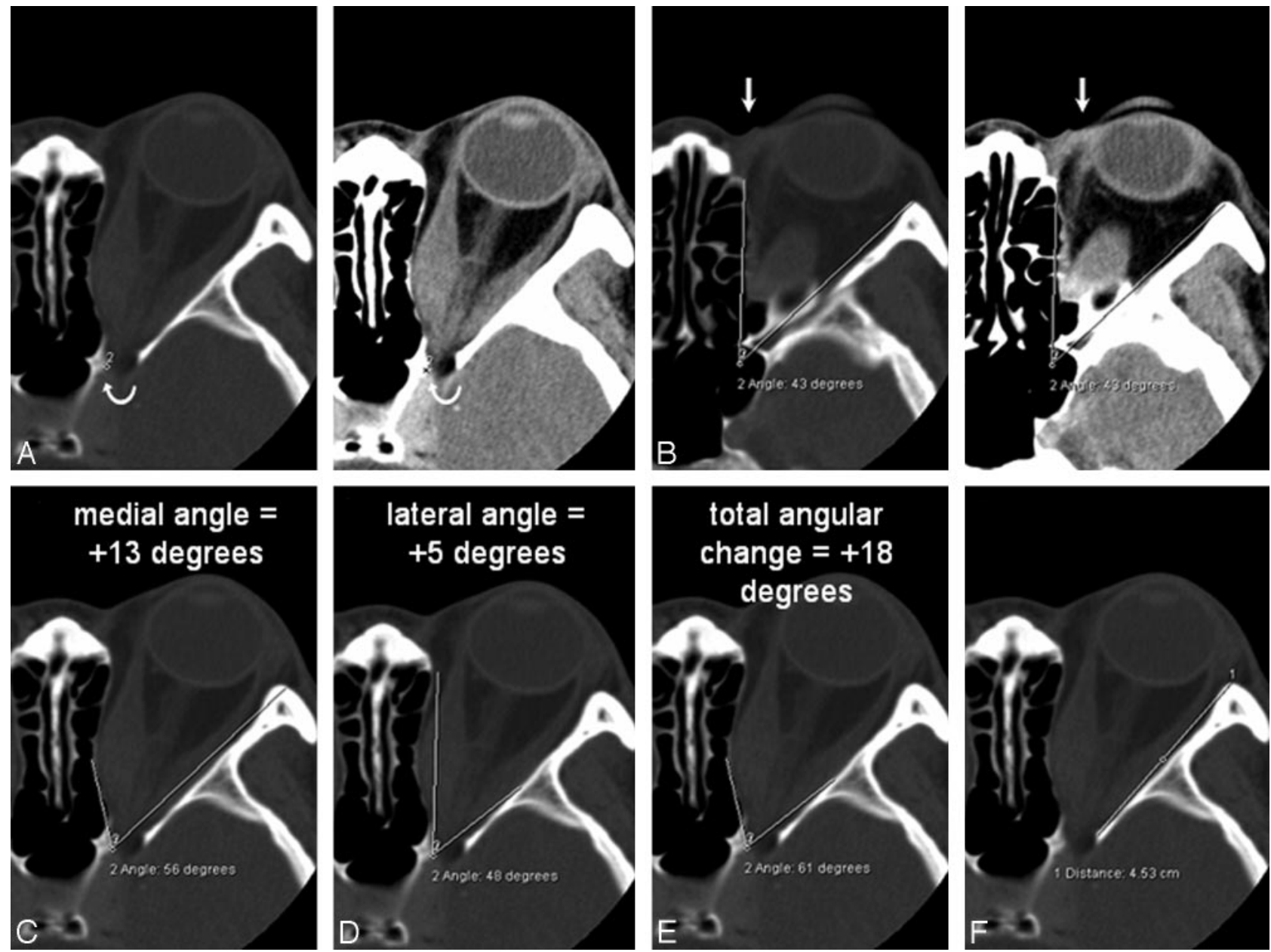

Fig 1. $A$ and $B$, Axial CT scans (bone window on left, soft tissue window on right) showing the orbital apex point indicated by the curved arrow $(A)$ and the orbital rim angle $(B)$. $A$, The apex point is defined as the anterolateral border of the groove in the sphenoid body formed by the intracavernous portion of the internal carotid artery (labeled 2) on the section just inferio to the anterior clinoid process. $B$, The orbital rim angle $\left(43^{\circ}\right)$ is measured at the level of the medial palpebral ligament (arrow). $C-E$, The same axial section containing the bulk of the medial and lateral rectus muscles shows the medial wall angle $(C)$, the lateral wall angle $(D)$, and the orbital apex angle $(E)$. For the medial and lateral walls, the angle that best describes the widest bony point of the orbital wall around the muscular bellies is recorded. The angular change is recorded as positive if the resultant angle is wider than the orbital rim angle, and negative for narrower angles. $F$, The length of the lateral orbital wall is measured on the section just inferior to the anterior clinoid.

tions before reading. The scans were read by a senior neuroradiologist (L.-L.C.) and a neuroradiology research associate (H.-E.T.) with approximately 15 and 2 years' practice, respectively. The readers were blinded to the presence of $\mathrm{ON}$ and independently reviewed the orbits at fixed soft-tissue and bone windows. One reader (H.-E.T.) repeated the measurements for 20 patients, 10 randomly selected from each patient group (40 orbits), on a separate occasion approximately a month apart.

\section{Quantitative CT Measurements}

The CT features of bony orbital confines, muscular enlargement, proptosis, and neurovascular structures were assessed quantitatively. Angular and linear measurements were made with the electronic calipers available on routine clinical reporting workstations.

\section{Bony Orbit}

The capacity of the bony apex was quantified by using standardized orbital angles on axial scans. The orbital apex was defined as the anterolateral border of the groove in the sphenoid body formed by the terminal intracavernous portion of the internal carotid artery (Fig $1 A)$. This apex point was electronically copied onto the series. The orbital rim angle was measured on the section containing the medial palpebral ligament (Fig 1B). This angle was used as a reference and was also electronically copied onto the series. The medial and lateral orbital wall angles (Fig $1 C,-D$ ) were measured as angular changes with respect to the orbital rim angle on the image containing the widest diameter of the medial and lateral rectus muscles. The angle that best described the widest bony point of the orbital wall around the muscular bellies was recorded. The angular change was recorded as positive if the resultant angle was wider than the orbital rim angle and negative for narrower angles. The total angular change was computed by the sum of the medial and lateral wall angles. The actual orbital apex angle (Fig 1E), as subtended by the medial and lateral orbital walls, was also recorded. The length of the lateral orbital wall was measured from the lateral border of the superior ophthalmic fissure to the orbital rim (Fig $1 F$ ).

For comparison, the above bony parameters were also examined in 12 patients ( 7 women) without Graves disease, 32-73 years of age (mean, 56 years), who had CT angiographic scans $(120 \mathrm{kVp}, 200 \mathrm{~mA}$, pitch of $0.9-1.2$ ) obtained for exclusion of aneurysm or vascular stenosis. Contiguous axial 3-mm sections (160-mm display FOV) parallel to the infraorbitomeatal line images were reconstructed from archived fine-section acquisitions for the measurements. There were no orbital abnormalities or skull base aneurysms in these patients. 


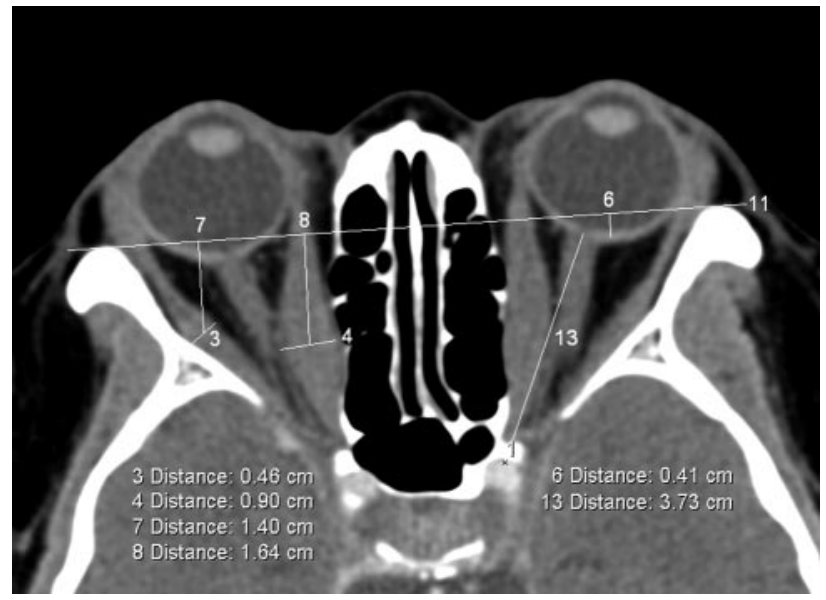

Fig 2. Axial section at midglobe level showing the interzygomatic line (labeled 11) and maximum horizontal diameters of the right medial and lateral rectus muscles (measurements labeled 4 and 3, respectively). The distance from the midpoint of the maximum muscular diameter of the medial (measurement 8 ) and lateral rectus muscles (measurement 7) to the interzygomatic line is also recorded. Proptosis of the left globe relative to the interzygomatic line is labeled as measurement 6. Left optic nerve stretch (labeled 13) is measured from the retrobulbar optic nerve to the orbital apex point (labeled 1).

\section{Soft-Tissue Structures}

The maximum horizontal, vertical, and inferolateral diameters of the medial and lateral recti (Fig 2), the inferior rectus and superior muscle group, and the superior oblique muscle, respectively, were measured on axial and coronal images by using the technique previously described by Nugent et al. ${ }^{8}$ These values were summated to yield the muscle diameter index (MDI) for each orbit. The perpendicular distance from the interzygomatic line to the midpoint of the maximum muscular diameter was also recorded on the axial images for the medial and lateral rectus muscles (Fig 2).

The degree of proptosis was measured with respect to the interzygomatic line, with negative and positive values for the posterior globe margin anterior and posterior to the interzygomatic line, respectively (Fig 2). The distance from the replicated orbital apex point to the retrobulbar optic nerve (on the axial section best depicting the latter) (Fig 2) was recorded as a measure of optic nerve stretch. The maximum diameter of the retrobulbar and midportions of the optic nerve sheath and the superior ophthalmic vein was measured on axial images according to the method described by Nugent et al. ${ }^{8}$

\section{Categoric Assessment}

The severity of optic nerve crowding was graded on coronal images, as described by Nugent et al. ${ }^{8}$ Grades $0,1,2$, and 3 refer to zero (none), $1 \%-25 \%$ (mild), 25\%-50\% (moderate), and >50\% (severe) effacement of perineural fat, respectively. The intracranial fat prolapse was recorded as present or absent by using the method described by Birchall et al. ${ }^{6}$

\section{Statistical Analysis}

Statistical analyses were performed by using the Statistical Package for the Social Sciences software, Version 10.1.3 (SPSS, Chicago, Ill). The intraclass correlation coefficient (ICC) was computed to assess interrater and intrarater variability. The Student 2-tailed $t$ test was used to compare the mean quantitative CT measurements between the 2 groups. For the categoric variables of apical crowding score and intracranial fat prolapse, the Mann-Whitney and Pearson $\chi^{2}$ tests were used for comparison, respectively. We also performed a stepwise multivariate logistic regression analysis, with disease status $(\mathrm{ON})$ as out- come and CT features as independent factors, adjusting for age group, and sex. The method of variable selection for entry of the factors and covariates into the logistic regression model was forward stepwise, with variable entry and exit criteria of $P<.05$ and $P>.10$, respectively. The odds ratio (OR) associated with each factor, the $95 \%$ confidence interval (CI) for the OR, and the $P$ value were determined. The sensitivity, specificity, and positive and negative predictive values from use of the logistic model in our dataset were also calculated. Statistical significance was defined at $P<.05$.

\section{Results}

The mean measurements, median grading score for optic nerve crowding, and count for intracranial fat prolapse were tabulated in Table 1. There were statistically significant associations (Table 1) between clinical $\mathrm{ON}$ and 1) bony angles $(P<.005)$, except the reference orbital rim angle; 2$)$ length of the lateral orbital wall $(P=.022) ; 3)$ muscular diameters and MDI $(P<.0005)$, except the superior oblique; 4$)$ medial rectus bulk from the interzygomatic line $(P<.05)$; and 5) apical crowding $(P<.0005)$. The comparison normative data for the bony measurements are tabulated in Table 2 .

The ICC for the bony angular measurements was excellent for inter-rater variability, ranging from 0.87 to 0.97 ; the ICC for intrarater variability was also very good, ranging from 0.84 to 0.98 . The ICC for the rest of the quantitative linear measurements and scores was also generally high for both interrater and intrarater variability, ranging from 0.81 to 0.99 .

The stepwise multivariate logistic regression analysis was performed to investigate the effect on the outcome of $\mathrm{ON}$ of each CT feature that was statistically discriminative between the 2 groups by univariate analysis at $P<.15$ (Table 1). Because age and sex could be confounding factors for optic neuropathy, adjustment for age $(\leq 50,>50$ years $)$ and sex was made. The MDI $(P<.0005$; OR $=1.653 ; 95 \% \mathrm{CI}$ for $\mathrm{OR}$, $1.304-2.096)$ and the medial $(P=.006 ; \mathrm{OR}=0.771 ; 95 \% \mathrm{CI}$ for OR, 0.642-0.927) and lateral $(P=.071 ; \mathrm{OR}=1.265 ; 95 \%$ CI for OR, 0.980-1.634) orbital wall angles were the only independent factors predictive of ON. Although the medial wall, lateral wall, and orbital apex angles increased linearly with the MDI in both patient groups, the relationship between the variables was clearly discrete in the 2 patient groups, as depicted graphically on the scatterplots in Fig 3. Within the borderline MDI range of $22-30 \mathrm{~mm}$ in Fig $3 B, 15$ of 26 patients with zero or negative medial angles $(57.7 \%)$ had ON $(P=.07)$, compared with 1 of 6 patients with positive medial angles (16.7\%).

From the logistic regression classification table of actualversus-predicted ON, the sensitivity, specificity, positive predictive value, and negative predictive value for the presence of $\mathrm{ON}$ by using MDI alone or in combination with the medial and lateral orbital wall angles are calculated (Table 3). Including the bony orbital angles improves the sensitivity to $73 \%$, specificity to $90 \%$, positive predictive value to $82 \%$, and negative predictive value to $85 \%$. The probability of ON, by using both the MDI and bony orbital angles for prediction, can be calculated by using the following logit function obtained from the logistic regression analysis:

Probability $(\mathrm{ON})=1 /(1+\exp [14.996-0.503 \mathrm{MDI}+$ $0.259 \mathrm{MW}-0.235 \mathrm{LW}])$ 


\begin{tabular}{|c|c|c|c|}
\hline Graves 0phthalmopathy & ON & Without ON & $P$ Value \\
\hline \multicolumn{4}{|l|}{ Demographics } \\
\hline No. orbits & 32 & 50 & \\
\hline Age & $55.9 \pm 11.5(39-78)$ & $44.7 \pm 11.0(28-65)$ & \\
\hline Sex (male, female) & $14 \mathrm{M}, 18 \mathrm{~F}$ & $20 \mathrm{M}, 30 \mathrm{~F}$ & \\
\hline \multicolumn{4}{|l|}{ Bony confines } \\
\hline \multicolumn{4}{|l|}{ Bony orbital angle (degrees) } \\
\hline Orbital rim & $42.4 \pm 2.5(39-49)$ & $42.2 \pm 2.4(37-51)$ & $<.727$ \\
\hline Medial wall & $-0.1 \pm 5.7(-8-12)$ & $-3.6 \pm 5.2(-13-12)$ & $<.006$ \\
\hline Lateral wall & $6.7 \pm 3.0(1-13)$ & $4.7 \pm 2.5(1-11)$ & $<.002$ \\
\hline Total angular change & $6.6 \pm 7.5(-3-20)$ & $1.1 \pm 6.5(-8-17)$ & $<.001$ \\
\hline Orbital apex & $49.0 \pm 8.2(38-65)$ & $43.3 \pm 7.4(30-59)$ & $<.002$ \\
\hline Length of lateral wall (mm) & $44.1 \pm 2.9(35.7-48.9)$ & $42.5 \pm 3.2(33.7-47.5)$ & $<.022$ \\
\hline \multicolumn{4}{|l|}{ Extraoccular muscles (mm) } \\
\hline \multicolumn{4}{|l|}{ Maximum muscle diameter } \\
\hline Medial rectus & $7.2 \pm 1.8(3.8-10.5)$ & $4.8 \pm 1.8(2.6-10.0)$ & $<.0005$ \\
\hline Lateral rectus & $4.9 \pm 1.5(2.3-8.2)$ & $3.8 \pm 0.9(2.1-6.4)$ & $<.0005$ \\
\hline Superior muscle group & $6.5 \pm 1.8(3.6-9.5)$ & $4.9 \pm 1.7(2.6-8.7)$ & $<.0005$ \\
\hline Inferior rectus & $7.1 \pm 2.0(3.2-12.8)$ & $5.0 \pm 1.6(2.6-9.5)$ & $<.0005$ \\
\hline Superior oblique & $3.0 \pm 0.7(1.8-4.5)$ & $2.8 \pm 0.7(1.8-4.4)$ & .212 \\
\hline $\mathrm{MDI}$ & $29.0 \pm 5.2(21.1-42.3)$ & $21.2 \pm 4.8(14.3-32.3)$ & $<.0005$ \\
\hline Medial rectus bulk from IZ line & $14.0 \pm 3.9(5.4-21.0)$ & $12.3 \pm 3.4(6.0-21.2)$ & .049 \\
\hline Lateral rectus bulk from IZ line & $16.0 \pm 3.0(10.8-21.2)$ & $14.8 \pm 2.8(10.7-21.1)$ & .084 \\
\hline \multicolumn{4}{|l|}{ Neurovascular structures } \\
\hline Proptosis from IZ line & $2.6 \pm 2.7(-3.5-7.0)$ & $3.5 \pm 4.0(-4.6-12.7)$ & .200 \\
\hline Optic nerve stretch & $40.8 \pm 3.2(35.0-47.9)$ & $39.7 \pm 4.1(30.7-46.6)$ & .243 \\
\hline \multicolumn{4}{|l|}{ Optic nerve sheath } \\
\hline Retrobulbar & $5.7 \pm 0.7(4.2-7.2)$ & $5.8 \pm 0.9(4.0-8.1)$ & .464 \\
\hline Waist & $3.7 \pm 0.6(2.4-5.2)$ & $3.6 \pm 0.7(1.6-5.5)$ & .703 \\
\hline Superior ophthalmic vein & $1.9 \pm 0.4(1.2-2.7)$ & $1.8 \pm 0.4(1.2-2.9)$ & .273 \\
\hline Apical crowding & $3(1-3)$ & $1(0-3)$ & $<.0005 \dagger$ \\
\hline Presence of intracranial fat prolapse & $11(34.4 \%)$ & $13(26.0 \%)$ & $.416 \ddagger$ \\
\hline
\end{tabular}

Note:- IZ line indicates interzygomatic line; ON, optic neuropathy; MDI, muscle diameter index.

* Descriptive statistics presented are mean \pm SD (range) and count (\%) for the presence of intracranial fat prolapse. All mean quantitative CT measurements between the 2 groups are compared using the Student 2-tailed $t$ test. Statistical significance is defined at $P<.05$

$\dagger$ The categoric variables are compared using the Mann-Whitney $U$ test. Statistical significance is defined at $P<.05$.

$\ddagger$ The categoric variables are compared using the $\chi^{2}$ square test. Statistical significance is defined at $P<.05$

\begin{tabular}{lc}
\hline \multicolumn{2}{l}{ Table 2: Normal bony orbital measurements } \\
\hline Bony Structure & orbits) \\
\hline Bony orbital angle (degrees) & Measurement \\
Orbital rim & $42.5 \pm 2.5(39-47)$ \\
Medial wall & $-4.6 \pm 1.9(-7$ to -1$)$ \\
Lateral wall & $4.9 \pm 2.5(1-10)$ \\
Total angular change & $0.3 \pm 2.9(-5-5)$ \\
Orbital apex & $42.8 \pm 4.2(35-52)$ \\
Length of lateral wall $(\mathrm{mm})$ & $42.7 \pm 1.9(39.4-45.9)$ \\
\hline
\end{tabular}

where MW refers to the medial wall angle and LW refers to the lateral wall angle.

\section{Discussion}

Although much work has been done evaluating the role of muscular enlargement and volumetric increase in intraorbital soft tissues in the pathophysiology of ON in Graves ophthalmopathy, the role of the bony orbit and its capacity on CT has not, to our knowledge, been previously investigated. There is wide variation in pneumatization of the sinonasal cavity. Well-pneumatized ethmoidal sinuses could encroach on and conceivably reduce the orbital apex space. Conversely, the medial orbital wall, being the thinnest orbital wall, would be readily remodeled medially toward the midline under raised intraorbital pressure, to make space for increased soft-tissue bulk in Graves ophthalmopathy. In addition, the depth of the bony orbit might also influence the rate of rise in orbital apex pressure and optic nerve stretch.

In this study, we attempted to characterize the bony orbit in quantitative measures readily available on standard clinical reporting workstations. We studied the angular capacity of the orbital apex on an axial plane because the orbital apex is normally taller than it is wide. Hence, we thought that the chief bony constraint under raised intraorbital pressure would lie on the axial rather than the sagittal plane. We found reliable bony reference points around the superior ophthalmic fissure despite the variable pneumatization of the sphenoid body. ${ }^{6}$ The mean, SD, and range of the orbital rim angle were highly comparable between the 2 patient groups (Table 1) and the normal comparison group (Table 2), suggesting the bony landmarks chosen to be effective reference points. The orbital apex angles were measured at the level of the medial and lateral rectus muscles because the extraocular muscles are chief occupants of the orbital apex in Graves ophthalmopathy and we believed that this would be the plane where maximal bony remodeling pressures would be exerted. This angular approach appeared to be reliable and reproducible, as suggested by our high ICC values.

In general, the medial wall angle (range, $25^{\circ}$ ) was more variable than the lateral wall angle $\left(14^{\circ}\right)$ in patients with 


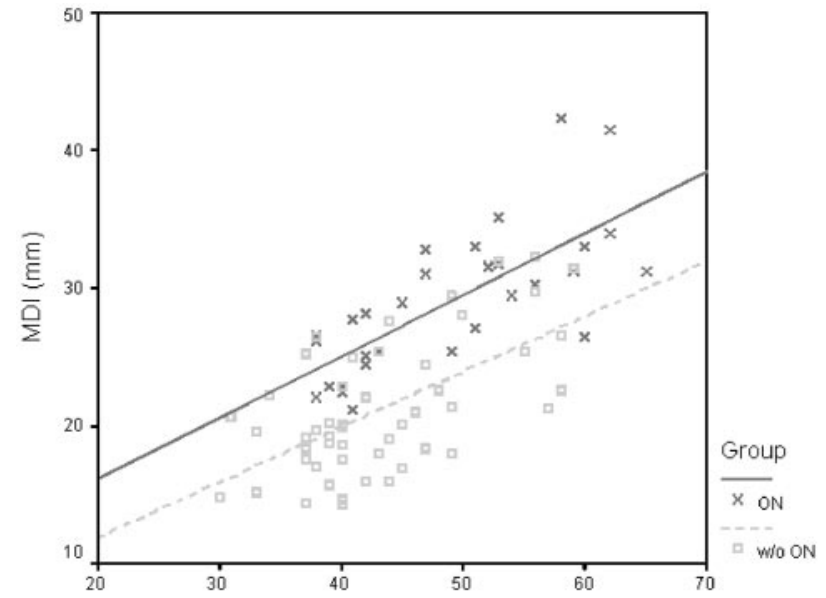

A

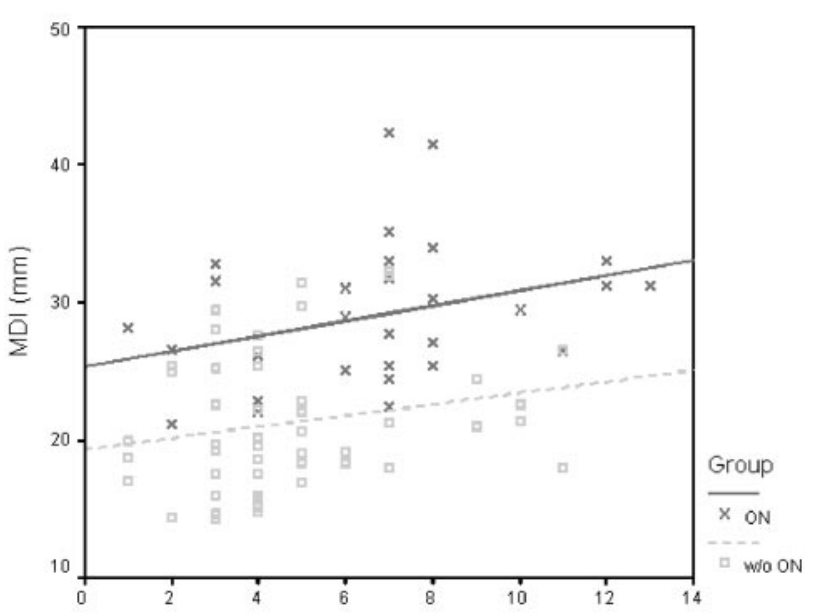

C

Lateral Orbital Wall Angle $\left({ }^{\circ}\right)$

Table 3: Sensitivity, specificity, and positive and negative predictive values of using MDI alone versus MDI in combination with medial and lateral orbital angles in predicting $\mathbf{O N}$ in Graves ophthalmopathy

\begin{tabular}{lcc}
\hline Percentage & MDI Alone & $\begin{array}{c}\text { MDI and Medial and } \\
\text { Lateral Wall Angles }\end{array}$ \\
\hline Sensitivity & $60.0(18 / 30)$ & $73.3(22 / 30)$ \\
Specificity & $86.0(43 / 50)$ & $90.0(45 / 50)$ \\
Positive predictive value & $72.0(18 / 25)$ & $81.5(22 / 27)$ \\
Negative predictive value & $78.2(43 / 55)$ & $84.9(45 / 53)$ \\
\hline
\end{tabular}

Graves ophthalmopathy because the thinner medial wall and air-filled ethmoidal sinuses offer less resistance to pressure remodeling compared with the thicker lateral wall. In comparison, the medial wall angle was less variable and always narrower than the orbital rim angle in the healthy group (Table 2 ). The mean bony orbital angles were wider in patients with ON and narrower in patients without (Table 1). Similarly, all mean muscular diameters were bigger in patients with $\mathrm{ON}$ than in those without (Table 1). Greater muscular enlargement is accompanied by wider bony orbital angles with or without $\mathrm{ON}$, and the relationship is the strongest for the medial wall angle (the steepest slope on the scatter plots in Fig 3). We believe that the wider angles in the ON group are a reflection of greater bony remodeling pressures generated by the higher MDIs found in this group. As can be seen from the OR

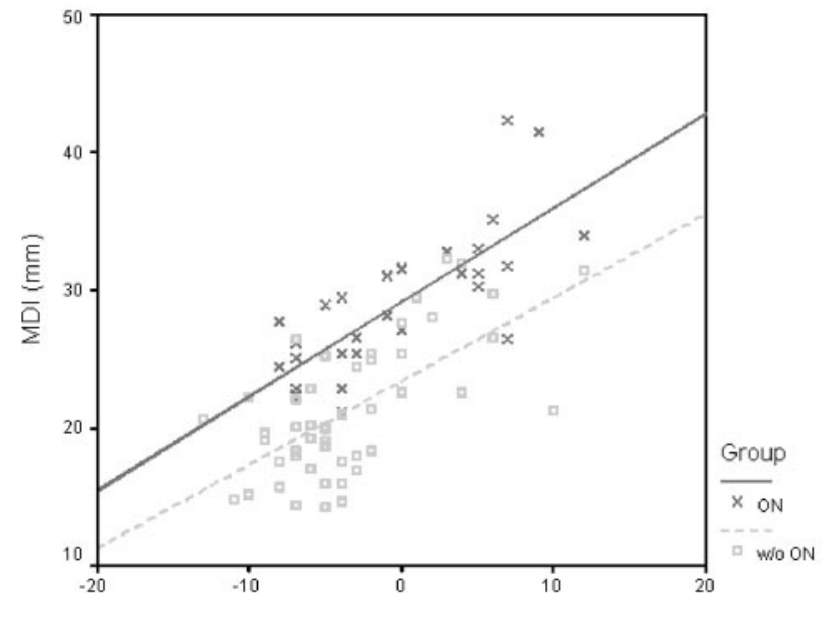

B

Medial Orbital Wall Angle $\left({ }^{\circ}\right)$

Fig 3. Scatterplots of the MDI and orbital apex angle $(A), \mathrm{MDI}$ and medial wall angle $(B)$, and $\mathrm{MDI}$ and lateral wall angle $(C)$ in patients with and without $\mathrm{ON}$. Greater muscular enlargement is accompanied by wider orbital angles with or without (w/0) ON. For identical MDI and orbital angles, the orbital angles are narrower and $\mathrm{MDI}$ greater in patients with $\mathrm{ON}$, respectively. Within the borderline MDI range of $22-30 \mathrm{~mm}$ in $B, 15$ of 26 patients with zero or negative medial angles $(57.7 \%)$ had $\mathrm{ON}(P=.07)$, compared with 1 of 6 patients with positive medial angles $(16.7 \%)$

obtained from the multivariate logistical regression analysis, the risk of $\mathrm{ON}$ increases with increases in MDI and lateral wall angles. However, when the medial wall angle increases, the risk of $\mathrm{ON}$ is, in fact, lower. Understanding this relationship aids the surgeon in the planning of orbital decompression, when this is considered as a treatment option.

What is noteworthy, for an identical MDI, the bony orbital angles were narrower in patients with $\mathrm{ON}$ and wider in patients without $\mathrm{ON}$ (lines representing the $\mathrm{ON}$ group are near parallel and higher than lines representing the group without ON, Fig 3 ). In the group without ON, there were 4 orbits with MDI bigger than the mean MDI in the ON group (Fig $3 A$ ). The orbital apex and medial wall angles in all 4 orbits were wider than the mean for the group with ON. Conversely for the same bony orbital angles, the MDI was bigger in patients with ON. We hypothesize that patients with ON may have congenitally narrower orbits or thicker orbital walls, which are less yielding to remodeling pressures, or a more acute disease onset preventing adequate bony reaction from occurring. Alternatively, the muscular enlargement may be so gross that the compensatory limits of bony remodeling are already surpassed.

The mean lateral orbital wall is longer in the ON group at $P=.022$ (Table 1), suggesting that a longer or deeper bony orbit might predispose to ON (Fig 4). In addition, the bulk of muscular enlargement (the point of maximum muscular en- 


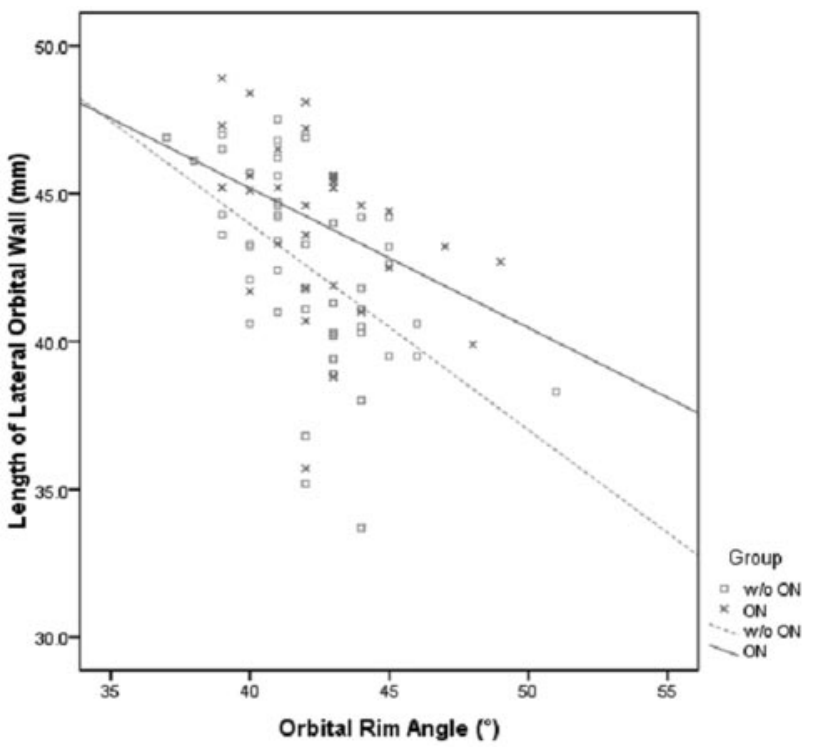

Fig 4. Scatterplot depicting the relationship between lengths of lateral orbital wall and orbital rim angles in patients with and without (w/0) ON.

largement) of the medial rectus muscle was nearer the orbital apex than the orbital rim (interzygomatic line) in the $\mathrm{ON}$ group $(P=.049$, Table 1$)$. This further supports the hypothesis that the $\mathrm{ON}$ in Graves ophthalmopathy results from mechanical compression on the optic nerve. Besides, previous histologic studies in patients with chronic Graves orbitopathy have demonstrated more pronounced axonal loss at the apical segments of the optic nerve. ${ }^{9}$ As previously reported by other investigators, ${ }^{4,5,8}$ we also found robust correlation between the degree of muscular enlargement and the presence of $\mathrm{ON}$ $(P<.0005$, Table 1$)$. However, the degree of muscular enlargement at which $\mathrm{ON}$ occurs is less clear.

The apical crowding score correlated strongly with clinical ON in our cohort $(P<.0005$, Table 1$)$. Several studies ${ }^{3,6-8}$ also found it to be a good diagnostic indicator of ON. However, it was not an independent predictor in our multivariate logistical regression analysis. Reports of $\mathrm{ON}$ in Graves ophthalmopathy occurring in the absence of pathologically enlarged extraocular muscles or apical crowding exist in the literature. ${ }^{5,7,10}$ Volumetric studies also tell us that there is an uncommon group of patients with Graves ophthalmopathy ( $8 \%$ in Forbes et $\mathrm{al}^{4}$ ) who only have increased fat volume, but normal muscle volume. ${ }^{4,11}$ Clearly risk prediction based solely on muscular enlargement or apical crowding scores is not enough. In our patients with $\mathrm{ON}$, there were 2 orbits with an apical crowding score of $\leq 1$. Both orbits had orbital apex and medial wall angles $\left(43\right.$ and $-4^{\circ}$ at an MDI of 25.4; 41 and $-4^{\circ}$ at an MDI of 21.1) narrower than the mean angle in the group without $\mathrm{ON}$ ( 43.3 and $-3.6^{\circ}$ at an MDI of 21.2). For the same increase in volume, solid attenuated muscle tissue would conceivably exert greater remodeling pressures than loose fatty tissue. Because bony remodeling compensates for raised intraorbital pressure and is protective, we postulate that patients who demonstrate only increased fat volume may be at risk for $\mathrm{ON}$ if their bony orbits are also inherently narrow. Further volumetric study correlating the intraorbital fat and extraoc- ular muscles with the orbital angles would confirm or refute the validity of this argument.

The degree of proptosis and optic nerve stretch was thought to indicate the severity of volumetric increase in orbital soft tissues. ${ }^{8}$ Although the ocular globes are more proptotic and the optic nerves are longer in patients with $\mathrm{ON}$, this finding was not significantly different from that in patients without ON (Table 1). Other groups have also reported a mixed picture for proptosis in relation to $\mathrm{ON}, 2,6,7,12,13$ and some have ascribed this to concomitant fibrosis or the tightness or laxity of orbital anatomy. ${ }^{13}$ Nugent et $\mathrm{al}^{8}$ suggested that the dimensions of the neurovascular structures could signify the degree of apical compression, but like others, ${ }^{6,7}$ we did not find these discriminative in our patient groups (Table 1). Some groups have found intracranial fat prolapse to be a good indicator of $\mathrm{ON},{ }^{6,7}$ whereas others report it to be an infrequent finding. ${ }^{3}$ Although intracranial fat prolapse was more common in our patients with $\mathrm{ON}$ (Table 1), its presence was not statistically significantly different between the 2 patient groups.

In conclusion, our study highlights the bony orbit and the medial orbital wall in particular as an independent predictor of ON in Graves ophthalmopathy. The capacity of the orbital apex is quantified on clinical reporting workstations, and the probability of $\mathrm{ON}$ is assessed by using the logit function obtained from logistic regression analysis. This approach could aid in the risk stratification of ON. Patients with a large MDI and a relatively narrow orbital apex and medial wall angles should undergo closer ophthalmic surveillance for early intervention. Similarly, the subgroup of patients with ON secondary to increased fat volumes in the absence of apical crowding may be identified. However, further large prospective studies would be useful to validate our findings.

\section{References}

1. Rootman J, Dolman P. Thyroid orbitopathy. In: Rootman J, ed. Diseases of the Orbit: A Multidiciplinary Approach. 2nd ed. Philadelphia: Lippincott Williams \& Wilkins; 2003:182-86

2. Trobe JD. Optic nerve involvement in dysthyroidism. Ophthalmology 1981;88:488-92

3. McKeag D, Lane C, Lazarus JH, et al. Clinical features of dysthyroid optic neuropathy: a European Group on Graves' Orbitopathy (EUGOGO) survey. Br J Ophthalmol 2007;91:455-58. Epub 2006 Oct 11

4. Forbes G, Gorman CA, Brennan MD, et al. Ophthalmopathy of Graves' disease: computerized volume measurements of the orbital fat and muscle. AJNR Am J Neuroradiol 1986;7:651-56

5. Barrett L, Glatt HJ, Burde RM, et al. Optic nerve dysfunction in thyroid eye disease: CT. Radiology 1988;167:503-07

6. Birchall D, Goodall KL, Noble JL, et al. Graves ophthalmopathy: intracranial fat prolapse on CT images as an indicator of optic nerve compression. Radiology 1996;200:123-27

7. Giaconi JA, Kazim M, Rho T, et al. CT scan evidence of dysthyroid optic neuropathy. Ophthal Plast Reconstr Surg 2002;18:177-82

8. Nugent RA, Belkin RI, Neigel JM, et al. Graves orbitopathy: correlation of CT and clinical findings Radiology 1990;177:675-82

9. Hufnagel TJ, Hickey WF, Cobbs WH, et al. Immunohistochemical and ultrastructural studies on the exenterated orbital tissues of a patient with Graves' disease. Ophthalmology 1984;91:1411-19

10. Anderson RL, Tweeten JP, Patrinely JR, et al. Dysthyroid optic neuropathy without extraocular muscle involvement. Ophthalmic Surg 1989;20:568-74

11. Trokel S, Kazim M, Moore S. Orbital fat removal: decompression for Graves orbitopathy. Ophthalmology 1993;100:674-82

12. Feldon SE, Muramatsu S, Weiner JM. Clinical classification of Graves' ophthalmopathy: identification of risk factors for optic neuropathy. Arch Ophthalmol 1984;102:1469-72

13. Kazim M, Trokel SL, Acaroglu G, et al. Reversal of dysthyroid optic neuropathy following orbital fat decompression. Br J Ophthalmol 2000;84:600-05 